\title{
Sparse correlation matching-based spectrum sensing for open spectrum communications
}

\author{
Eva Lagunas ${ }^{*}$ and Montse Nájar ${ }^{1,2}$
}

\begin{abstract}
To deal with the current spectrum scarcity problem and exploiting the fact that exclusive access through tightly regulated licensing leads to idle spectrum, cognitive radio has been proposed as a way to reuse this underutilized spectrum in an opportunistic manner, i.e., allowing the use of temporarily unused licensed spectrum to secondary users who have no spectrum licenses. To protect the licensed users from the cognitive users' interference, the opportunistic user requires knowledge of the original license holder activity. In this article, a feature-based approach for spectrum sensing based on periodic non-uniform sampling is addressed. In particular, we face the compressed-sampling version of detecting predetermined spectral shapes in sparse wideband regimes by means of a correlation-matching procedure.
\end{abstract}

Keywords: cognitive radio, compressed sensing, spectrum sensing, correlation matching

\section{Introduction}

Current spectrum division among users in wireless communication systems is assigned by regulatory and licensing bodies like the Federal Communication Commission ${ }^{\text {a }}$ (FCC) in the US or the European Telecommunications Standard Institute ${ }^{\mathrm{b}}$ (ETSI) in Europe. In the usual spectrum management approach, the radio spectrum is divided into fixed and non-overlapping blocks, which are assigned to different services and wireless technologies. The recent proliferation of wireless communications services together with the inflexible spectrum regulations have resulted in a crowded radio frequency (RF) spectrum. This spectrum congestion becomes a bottleneck for the increasing demand of new transmission bands, which can rarely be satisfied using permanent allocation.

The scarcity of electromagnetic spectrum is obvious, but the real problem is not a dearth of radio spectrum; it's the way that spectrum is used. The radio spectrum is actually poorly utilized in many bands in the sense that large portion of the assigned bands are not used most of the time [1]. A solution to this inefficiency is to allow opportunistic unlicensed access to the poorly utilized frequency bands that have been already allocated.

\footnotetext{
* Correspondence: eva.lagunas@upc.edu

'Department of Signal Theory and Communications, Technical University of Catalonia (UPC), c/Jordi Girona 1-3, 08034, Barcelona, Spain Full list of author information is available at the end of the article
}

This more flexible allocation approach is known as cognitive radio (CR) [2].

In $\mathrm{CR}$, radios opportunistically look for holes (non-used spectrum gaps) in the licensed spectrum, which can subsequently be exploited for setting up a communication link. However, the approach described previously requires knowledge of the primary (licensed) user spectrum activity in order to avoid causing interference. Protecting the noncognitive users is mandatory, since they have the priority of service. The task of accurately detecting the presence of licensed user is encompassed in spectrum sensing. The signal-processing fundamentals specific to spectrum sensing implementation have been investigated in [3]. Among the implementation challenges mentioned in [3], the most critical design problem is the need to process very wide bandwidth (regardless of operating frequency range) and reliably detect presence of primary users. Identifying unoccupied frequencies is a complicated problem which involves sampling many points on the radio spectrum. Moreover, with the current analog-to-digital converters (ADC) technology, wideband RF signal digitising is a quite demanding task. Consequently, each CR node can only sense a relatively narrow band.

Sampling at the Nyquist rate is shown to be inefficient when the signals of interest contain only a small number of significant frequencies relative to the bandlimit [4]. To alleviate the sampling bottleneck, a promising 
alternative for this type of sparse signals is the use of sub-Nyquist sampling techniques.

\subsection{Background and prominent related work}

This article blends two topics: Spectrum sensing and sub-Nyquist sampling. The goal of this section is to present a review of the most prominent published works related to spectrum sensing and sub-Nyquist sampling techniques.

\subsubsection{Beyond Nyquist sampling rate}

Signal acquisition is a main topic in signal processing. Sampling theorems provide the bridge between the continuous and the discrete-time worlds. The most famous theorem is often attributed to Shannon [5,6] (but usually called Nyquist rate) and says that the sampling rate must be twice the maximum frequency present in the signal in order to perfectly recover the signal. However, sampling at twice as high as the upper frequency of the signal spectra might be problematic when the band limit of the signal is large. The usual method of sampling at equally spaced instants of time permits unambiguous reconstruction of the original signal if and only if the spectra of the signal is known in advance to lie in the Nyquist band.

Shapiro and Silverman [7] were the first who noticed these problems back in 1960. In order to avoid aliasing they proposed unequally spaced instants of sampling time. In fact, they showed that random sampling schemes succeed in eliminating aliasing, while others do not. One example proposed in [7] was to take the sampling time the occurrence times of the events of some Poisson process.

Later in the 1970s, Beutler [8] generalized the formulation of the alias-free sampling problem and studied special cases depending on the spectral distribution of the signals. In this context, Masry [9] studied the random sampling in a more general framework. In the 1990s, Bilinskis [10] presented his breakthrough study in digital alias-free signal processing (DASP) which was summarized in 2005 in a book with the same name [11]. As the term suggests, DASP is focussed on the problem of aliasing prevention, as well as all the previous mentioned methods.

All these researchers realized that the restrictions defined by Shannon-Nyquist do not have to be always satisfied. Of course, the obvious way (and the simplest way) to avoid aliasing when there is no extra information available is to require two times the maximum frequency present in the signal. This approach is very conservative but ensures perfect recovery of the signal. The strong requirements of the ADCs can be reduced by exploiting prior knowledge on the signal model. Due to the low occupancy of many communication systems, whose frequency support is much smaller than the band limit, the spectrum can be considered sparse and the uniform sampling becomes very redundant.

Following this vision, a clever way of sampling the signal is the periodic non-uniform sampling. This method, called multi-coset sampler and originally proposed by Feng and Bresler [12], shares many aspects with the recent compressed sensing (CS) theory. CS $[13,14]$ provides a robust framework for reducing the number of measurements required to summarize sparse signals allowing to compress the data while is sampled. Although multi-coset sampling can be casted into a CS framework, its implementation becomes simpler: while usually CS considers an analog to Information converter (AIC), in the multi-coset approach only a limited number of parallel ADCs operating at low sampling rate are needed. In this context, Mishali and Eldar [15] proposed a sub-Nyquist analog-to-digital converter of wideband inputs, the first reported wideband hardware for subNyquist conversion based on the multi-coset technique (as the authors claim).

\subsubsection{Spectrum sensing}

A CR monitors the available spectrum bands, captures their information, and then detects the spectrum holes where is possible to transmit in an opportunistic manner in order to avoid possible interferences with the primary or licensed users.

The identification procedure of available spectrum is quite a difficult task due to the strict requirements imposed to guarantee no harmful interference to the licensed users. In general, the minimum signal-to-noise ratio (SNR) at which the primary signal may still be accurately detected required by the sensing procedure is very low. Thus, low SNR levels must be sensed which translates into a high detection sensitivity.

A second constraint is the required detection time [16]. The longer the time that we sense, the better the signal processing gain. However, the spectrum behaves dynamically, changing all the time, and cognitive users need to be aware of these fast changes. Another desirable feature is that the primary user detector has to provide an accurate power level for the primary user. The estimated power level can be used to obtain information about the distance at which the primary user is located providing the level of interference that unlicensed users represent.

A number of different methods are proposed for primary user detection. According to the a priori information required to detect the primary user and the resulting complexity and accuracy, general spectrum sensing techniques can be categorized in the following types: blind sensing and feature-based sensing techniques. One of the most popular blind detection strategy is energy detector (ED) [17]. However, ED is unable to discriminate between the sources of received energy. On the other 
hand, the most famous feature-based method is the matched filter. If the full structure of the primary signal is known (together with time and carrier synchronization), the optimal detector is the matched filter detector. Unfortunately, the complete knowledge of the primary signal is not usually available. If only some features of the primary signal are known, feature-based detectors such cyclostationary detector [18] are more suitable. In feature-based approaches, the secondary users are considered as interference. A survey of the most common spectrum sensing techniques, both non-feature and feature-based detectors, has been published in [19].

As it was mentioned before, the design of the analog front-end is critical in the case of CR. The worst problem is the high sampling rate required to process very wide bandwidth. The present literature for sparse spectrum sensing is still in its early stages of development. The traditional way for detecting holes in a wide-band spectrum is channel-by-channel scanning. In order to implement this, an RF front-end with a bank of tunable and narrow bandpass filters is needed. Some alternative methods have been proposed in the literature to facilitate the wide-band sensing process [16,20,21]. In [16], a compressive sensing approach is used to reconstruct the spectrum of a wide-band signal using time samples, which studies for special signals whose Fourier transform is real. In [20], the received analog signal is sampled at the information rate of the signal using an AIC. An estimate of the original signal spectrum is then made based on CS reconstruction using a wavelet edge detector. Wang et al. [21] proposed a two-step compressed spectrum sensing method which first quickly estimates the actual sparsity order of the wide spectrum of interest, and adjusts the total number of samples collected according to the estimated signal sparsity order.

\subsection{Outline and contributions}

Many research studies such as Viberg [22] or Lexa [23] use the sub-Nyquist methods to obtain information of the unknown power spectrum from the compressed samples avoiding the signal reconstruction. In particular, in [23], the estimator does not require signal reconstruction and can be directly obtained from a straightforward application of nonnegative least squares. In [22], the estimation of the signal spectrum is skipped, and the occupied channels are directly detected from the sampled data in the time domain. Others such as Giannakis [16] or Leus [20] look for an estimate of the original signal spectrum based on CS reconstruction using a wavelet edge detector. Here, a more particular problem is studied. In this article, the problem of detecting predetermined spectral shapes present in the spectrum of the wide-band signal received at the CR detector is addressed. The final goal of this proposal is to determine the spectrum occupancy of the licensed system. Taking advantage of the sparsity of the signals sent out over the spectrum, a sub-Nyquist periodic non-uniform sampling is used to reduce the amount of data needed to find the white space and still maintain a high degree of accuracy.

The procedure is developed following a correlation matching framework, changing the traditional single frequency scan to a spectral scan with a particular shape. The spectrum sensing scheme considered here was first presented in [24] without solving the sampling bottleneck. In [24], the data autocorrelation matrix was estimated from the Nyquist samples of the analog received signal due to the traditional assumption that the sampling state needs to acquire the data at the Nyquist rate, corresponding to twice the signal bandwidth. There are two drawbacks in [24]: (1) due to the timing requirements for rapid sensing, only a limited number of measurements can be acquired from the received signal; and (2) the implementation quickly becomes untenable for wideband spectrum sensing. Here, we take advantage of the sparsity of the spectrum to alleviate the sampling burden. Sensing and compressing in a single stage allows fast spectrum sensing while simplifying the implementation. In this article, the estimate of the data autocorrelation matrix is directly obtained from the compressed samples. Three procedures are derived depending on the criteria used to compare the estimated matrix with the predetermined one. We evaluate the resulting detector with particular examples, we derive simulated ROCs and the performance is evaluated with the RMSE and compared with classical filter-bank approaches as well as with the non-compressed version of the procedure.

This article is organized as follows. The following section states the signal model and problem formulation introducing the periodic sub-Nyquist sampling notation. Then, the following section introduces the spectrum sensing method paying special attention to the data autocorrelation matrix estimation. Finally, the last section shows the simulation results and the performance evaluation. The concluding remarks of this article are given in the very last section.

\section{Signal model, definitions and problem statement}

We consider a wideband signal $x(t)$ which may represent the superposition of different primary services in a CR network. This signal is assumed to be multi-band signal, i.e, a bandlimited, continuous-time, squared integrable signal that has all of its energy concentrated in one or more disjoint frequency bands.

Denoting the Fourier transform of $x(t)$ as $X(f)$, the spectral support $F \subset\left[0, f_{\max }\right]$ of the multiband signal $x$ $(t)$ is the union of the frequency intervals that contain the signal's energy: 


$$
F=\bigcup_{i=1}^{N}\left[a_{i}, b_{i}\right)
$$

A sparse multiband signal is thus a multiband signal whose spectral support has Lebesgue measure that is small relative to the overall signal bandwidth [25]. To this end, the spectral occupancy $\Omega$ is defined as,

$$
\Omega=\frac{\lambda(F)}{f_{\max }} \quad 0 \leq \Omega \leq 1
$$

where $\lambda(F)$ is the Lebesgue measure of the frequency set $F$ which, in this particular case, is equal to $\sum_{i=1}^{N}\left(b_{i}-a_{i}\right)$. For the set of sparse multiband signals $\Omega$ ranges from 0 to 0.5 (see Figure 1). In the spectrum sensing framework, the spectral support $F$ is unknown but the total bandwidth under study is assumed to be sparse.

The goal of this article is to obtain the frequency locations and the power levels of the primary users using a correlation matching spectrum sensing strategy based on the compressed samples obtained with a periodic non-uniform sub-Nyquist sampling. The diagram of the cognitive receiver is sketched in Figure 2. First, taking advantage of the sparsity of the received signal spectrum, a multi-coset sampling is used to overcome the problem of high sampling rate. Then, the compressed samples are processed in the autocorrelation estimation stage and finally, the correlation-matching based spectrum sensing is performed using a predetermined spectral shape, which has to be known a priori.

\section{Sparse-based sample acquisition}

In multi-coset sampling, we first pick a suitable sampling period $T$. The inverse of this period $(1 / T)$ will determine the base frequency of the system, being $1 / T$ at least equal to the Nyquist rate so that sampling at 1/ $T$ ensures no aliasing. Given the received multiband signal $y(t)$, the periodic nonuniform samples are obtained at the time instants,

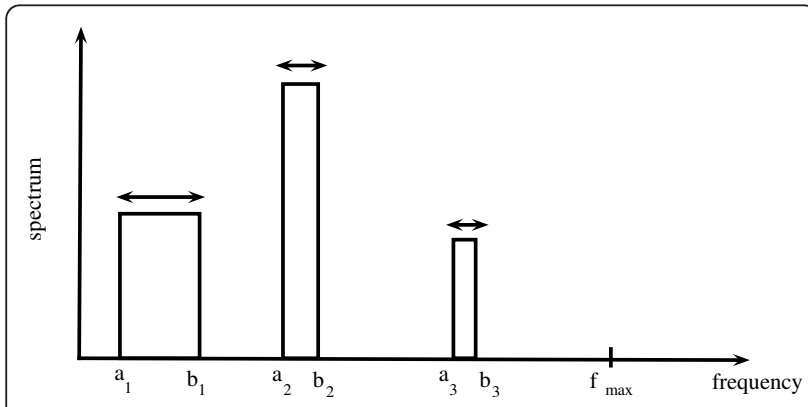

Figure 1 Spectrum of a sparse multiband signal, with $\mathrm{N}=3$ and spectral occupancy $\Omega$ lower than 0.5 .

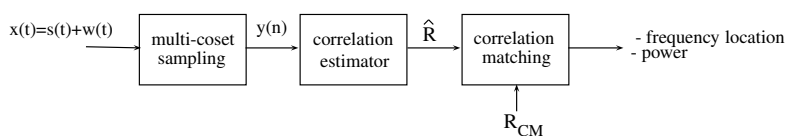

Figure 2 Block diagram of the cognitive receiver: multi-coset sampling followed by the correlation estimator and the proposed correlation-matching based spectrum sensing technique.

$$
t_{i}(n)=\left(n L+c_{i}\right) T
$$

where $L>0$ is a suitable integer, $i=1,2, \ldots, p$ and $n \in$ $\mathbb{Z}$. The set $\left\{c_{i}\right\}$ contains $p$ distinct integers chosen from $\{0,1, \ldots, L-1\}$. The reader can notice that the multi-coset sampling process can be viewed as a classical Nyquist sampling followed by a block that discards all but $p$ samples in every block of $L$ samples periodically. The samples which are not thrown away are specified by the set $\left\{c_{i}\right\}$.

Thus, a sequence (or coset, hence the name of the method) of equally-spaced samples is obtained for each $c_{i}$. The period of each one of these sequences is equal to $L T$. Therefore, one possible implementation consists of $p$ parallel ADCs, each working uniformly with period $L T$. Another widely-used notation for the multi-coset sampling is to express each $i$ th sampling scheme as follows,

$$
y^{i}[n]= \begin{cases}y(n T), & n=m L+c_{i}, \quad m \in \mathbb{Z} \\ 0, & \text { Otherwise }\end{cases}
$$

where $y(t)$ denotes the received signal, which contains the multiband signal $x(t)$, plus an interference $i(t)$, plus a double-side complex zero-mean AWGN $w(t)$ with spectral density $N_{0} / 2$,

$$
y(t)=x(t)+i(t)+w(t)
$$

The interference is assumed independent of the noise and desired signal, and its spectral shape is different from that of the desired.

The set $\left\{c_{i}\right\}$ is referred to as an $(L, p)$ sampling pattern and the integer $L$ as the period of the pattern. Figure 3 shows a scheme of how the $p$ cosets are obtained.

The complete observation consists of a data record of $M$ blocks of $p$ nonuniform samples notated as $\mathbf{y}_{m}$. Thus, the notation can be compacted in $\mathbf{Y}$ as follows,

$$
\mathbf{Y}=\left[\mathbf{y}_{1} \ldots \mathbf{y}_{M}\right]
$$

The sub-Nyquist data matrix $\mathbf{Y}$ has dimension $p \times M$.

3.1 Relation between multi-coset sampling and CS theory To translate the multi-coset sampling notation into a CS notation, let us consider $\mathbf{z}_{m}$ as the $m$ th block of $L$ 


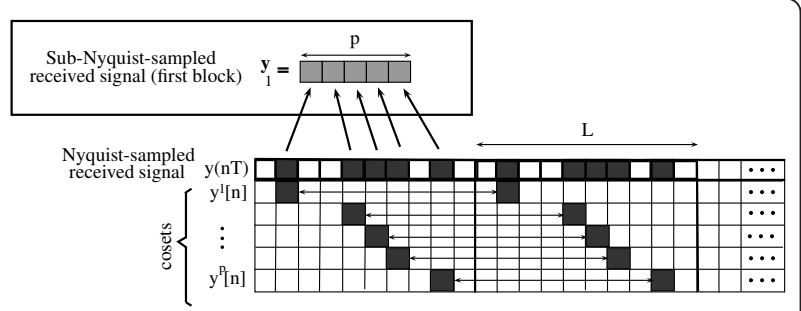

Figure 3 Scheme of the multi-coset generation. $L=10$ and $p=$ 5 .

uniform Nyquist samples of $y(t)$,

$$
\mathbf{z}_{m}=\left[\gamma\left(t_{1}^{m}\right) \ldots \gamma\left(t_{L}^{m}\right)\right]^{T}
$$

where $t_{n}^{m}=(m L+n) T$. As we know, this signal is sparse if it is expressed in the frequency domain. The implication of sparsity in the novel CS theory is that one can discard the part of the coefficients without much perceptual loss. Let us now consider the classical linear measurement model for the above block,

$$
\mathbf{y}_{m}=\Phi_{m} \mathbf{z}_{m}
$$

The problem in CS consist of designing a convenient measurement matrix $\Phi_{m}$ such that salient information in any compressible signal is not damaged by the dimensionality reduction. A low value of coherence between $\Phi_{m}$ and the basis where the signal becomes sparse (Fourier in our case) is desirable in order to ensure mutually independent matrices and therefore better compressive sampling. The incoherence is defined as the maximum value amongst inner product of the orthonormal basis where the signal becomes sparse, and the orthonormal measurement matrix $\Phi_{m}$. In our example, the maximal incoherence associated to the Fourier basis is given by the canonical or spike basis $\phi_{k}(t)=\delta(t$ $-k)$. Thus, $\Phi_{m}$ must be a matrix that randomly selects $p$ samples of $\mathbf{z}_{m}$, where $p<L$. This matrix $\Phi_{\mathrm{m}}$ is given by randomly selecting $p$ rows of the identity matrix $\mathbf{I}_{L}$.

If matrix $\Phi_{m}$ is obtained from the identity matrix and it remains the same whatever the $m$ th block is considered, then the signal $\mathbf{y}_{m}$ is the same than the one obtained using the multi-coset sampling. As the matrix notation is much more clear, we will proceed following this notation,

$$
\mathbf{Y}=\left[\mathbf{y}_{1} \ldots \mathbf{y}_{M}\right]=\left[\Phi \mathbf{z}_{1} \ldots \Phi \mathbf{z}_{M}\right]
$$

Following the notation of (8), the correlation matrix $\hat{\mathbf{R}}_{y} \in \mathbb{C}^{p \times p}$ can be obtained as,

$$
\hat{\mathbf{R}}_{y}=\frac{1}{M} \sum_{m=1}^{M} \mathbf{y}_{m} \mathbf{y}_{m}^{H}=\Phi\left[\frac{1}{M} \sum_{m=1}^{M} \mathbf{z}_{m} \mathbf{z}_{m}^{H}\right] \Phi^{H}=\Phi \hat{\mathbf{R}}_{x} \Phi^{H}+\tilde{\mathbf{R}}_{i}+\tilde{\mathbf{R}}_{w}
$$

where $\hat{\mathbf{R}}_{x} \in \mathbb{C}^{L \times L}$ indicates the estimated autocorrelation matrix of the primary user that we want to detect, and $\tilde{\mathbf{R}}_{i} \in \mathbb{C}^{p \times p}$ and $\tilde{\mathbf{R}}_{w} \in \mathbb{C}^{p \times p}$ denotes the sub-Nyquist interference and the sub-Nyquist noise estimated autocorrelation matrix, respectively. As $\Phi$ comes from the identity matrix, $\tilde{\mathbf{R}}_{w}$ is expected to be $\sigma^{2} \mathbf{I}_{p}$. This notation simplifies the notation presented in [22], where the coset samples are fractional shifted and used to compute the correlation matrix of the signal.

\section{Sparse correlation matching-based spectrum sensing}

The proposed procedure consists of detecting the presence of a licensed user whose power spectral shape (called candidate spectral shape henceforth) is the only prior knowledge we have. Based on a feature-based detector perspective, a correlation matching approach is used with the candidate spectral shape as a reference. The baseband candidate autocorrelation matrix $\mathbf{R}_{b}$, which depends only on the basic pulse used by the modulation transport, can be easily obtained from the candidate spectrum shape.

Therefore, in order to obtain the frequency location of each primary user, the candidate autocorrelation $\mathbf{R}_{b}$ is modulated by a rank-one matrix formed by the steering frequency vector at the sensed frequency $w$ as follows,

$$
\mathbf{R}_{c m}=\left[\mathbf{R}_{b} \odot \mathbf{s s}^{H}\right]
$$

where $\odot$ denotes the elementwise product of two matrices, $\mathbf{s}=\left[1 e^{j w} \ldots e^{j(L-1) w}\right]^{T}$. Note that in (11) the dependency on $w$ has been removed to clarify notation.

According to (11) and assuming one active primary user, the corresponding model for the data autocorrelation matrix defined in (10) is given by,

$$
\mathbf{R}_{y}=\gamma\left(w_{s}\right) \Phi \mathbf{R}_{c m} \Phi^{H}+\mathbf{R}_{n}
$$

where $\mathbf{R}_{n}$ is the randomly sampled AWGN plus interference autocorrelation matrix and $\gamma\left(w_{s}\right)$ is the power level at frequency $w_{s}$, which denotes the tentative frequency of the active primary user.

Summarizing, the problem to solve consists in finding the frequency that the compressed candidate correlation has to be modulated to best fit the data autocorrelation matrix $\hat{\mathbf{R}}_{y}$ and to find the contribution of this modulated candidate autocorrelation contained in $\hat{\mathbf{R}}_{y}$. Thus, the procedure not only provides the frequency location of the desired user but also an estimation of its transmitted power.

Based on these assumptions, an estimate of the power level $\gamma$ can be formulated as, 


$$
\min _{\gamma} \Psi\left(\hat{\mathbf{R}}_{\gamma}, \gamma \Phi \mathbf{R}_{c m} \Phi^{H}\right)
$$

where $\Psi(\cdot, \cdot)$ is an error function between the two matrices. Note that the solution to (13) will be clearly a function of the steering frequency.

The different estimates result from the proper choice of the aforementioned error function can be divided in two groups: (1) error functions based on the distance between the two matrices and (2) error functions based on the positive definite character of the difference $\left(\hat{\mathbf{R}}_{y}-\gamma \Phi \mathbf{R}_{c m} \Phi^{H}\right)$.

\subsection{Derivation of different methods depending on the choice of $\Psi(\bullet, \bullet)$}

Three different candidate methods were defined in [24] for the non-compressed case. Here comes a brief review of the three procedures adapted to the sparse signal acquisition case.

The first one is a detector based on the traditional Euclidean metric (Frobe-nius norm of the difference between matrices) and is denoted as CANDIDATE-F. Thus, our problem can be written as,

$$
\min _{\gamma}\left|\hat{\mathbf{R}}_{\gamma}-\gamma \Phi \mathbf{R}_{c m} \Phi^{H}\right|_{F}
$$

and the solution to (14) is given by,

$$
\gamma_{F}=\frac{\operatorname{Trace}\left(\Phi \mathbf{R}_{c m} \Phi^{H} \hat{\mathbf{R}}_{y}\right)}{\operatorname{Trace}\left(\left(\Phi \mathbf{R}_{c m} \Phi^{H}\right)^{2}\right)}
$$

However, this estimate does not preserve the positive definite property of the difference.

The second alternative is a detector based on the geodesic distance (CANDIDATE-G) that best suits the space generated by hermitian matrices. The set of autocorrelation matrices is a convex cone because they are hermitian and positive semidefinite matrices. Therefore, a more proper distance for the space generated by the semidefinite positive matrices is the geodesic distance. The geodesic distance between $\mathbf{R}_{1}$ and $\mathbf{R}_{2}$ is given by,

$$
d_{g e o}^{2}\left(\mathbf{R}_{2}, \mathbf{R}_{1}\right)=\sum_{q=1}^{Q}\left(\operatorname{Ln}\left(\lambda_{q}\right)\right)^{2}
$$

where

$$
\mathbf{R}_{1}^{-1} \mathbf{R}_{2} \mathbf{e}_{q}=\lambda_{q} \mathbf{e}_{q} \text { for } q=1, \ldots, Q
$$

Identifying $\mathbf{R}_{1}=\gamma \Phi \mathbf{R}_{c m} \Phi^{H}$ and $\mathbf{R}_{2}=\hat{\mathbf{R}}_{y}$ and minimizing (16), the power level estimate and the resulting minimum geodesic distance can be derived (18).

$$
\begin{aligned}
& \gamma_{G}=\left(\prod_{q=1}^{Q} \lambda_{q}\right)^{\frac{1}{Q}} \\
& d_{g e o, \min }^{2}=\sum_{q=1}^{Q}\left|\ln \left(l_{q}\right)\right|^{2}=\sum_{q=1}^{Q}\left|\ln \left(\lambda_{q} / \gamma_{G}\right)\right|^{2}
\end{aligned}
$$

where $\lambda_{q}(q=1, \ldots, Q)$ denotes the $Q$ generalized eigenvalues of the pair $\left(\hat{\mathbf{R}}_{y}, \Phi \mathbf{R}_{c m} \Phi^{H}\right)$. That is,

$$
\begin{aligned}
& \hat{\mathbf{R}}_{\gamma} \mathbf{e}_{q}=\lambda_{q} \Phi \mathbf{R}_{c m} \Phi^{H} \mathbf{e}_{q} \text { for } q=1, \ldots, Q \\
& \frac{1}{\gamma}\left(\Phi \mathbf{R}_{c m} \Phi^{H}\right)^{-1} \hat{\mathbf{R}}_{\gamma} \mathbf{e}_{q}=l_{q} \mathbf{e}_{q} \text { for } q=1, \ldots, Q
\end{aligned}
$$

In interesting, the power level estimate $\gamma_{G}$ does not depend on the frequency $w$ of the candidate. Thus, the power level estimate $\gamma_{G}$ does not require frequency scanning. The frequency location is obtained detecting the maximum of the inverse of the minimum geodesic distance $(18 \mathrm{~b})$ versus frequency.

Finally, a third power level estimate can be derived by forcing a positive definite difference between the data autocorrelation matrix and the candidate matrix, as it was done in [24]. Here, we propose a different way to get to the same result following a minimum mean square error between the received signal and the candidate signal.

The received signal model can be simplified as,

$$
\mathbf{y}=\sqrt{\gamma} \mathbf{x}+\mathbf{n}
$$

where $\mathbf{x}$ denotes here the candidate signal, $\sqrt{\gamma}$ its amplitude and $\mathbf{n}$ the noise plus interference.

Let us define a filter $\mathbf{A}$, which is applied to the received signal $\mathbf{y}$ in order to obtain an approximation of the desired signal $\mathbf{x}$. The resulting error $\mathbf{e}$ is defined as,

$$
e=x-A y
$$

The covariance matrix of the aforementioned error,

$$
\xi=E\left\{\mathbf{e} \mathbf{e}^{H}\right\}=\mathbf{R}_{x x}-\mathbf{R}_{x y} \mathrm{~A}^{H}-A \mathbf{R}_{y x}+A \mathbf{R}_{y y} A^{H}
$$

Given that $\quad \mathbf{R}_{x y}=\mathbf{R}_{y x}=\sqrt{\gamma} \mathbf{R}_{x x} \quad$ and $\nabla_{A^{H}} \xi=A \mathbf{R}_{y y}-A \mathbf{R}_{x y}$, the optimal filter and the minimum error are given by,

$$
\begin{aligned}
& \mathbf{A}_{o p}=\sqrt{\gamma} \mathbf{R}_{x x} \mathbf{R}_{y y}^{-1} \\
& \xi_{\text {min }}=\mathbf{R}_{x x}\left(\mathbf{I}-\gamma \mathbf{R}_{y y}^{-1} \mathbf{R}_{x x}\right)
\end{aligned}
$$


Matrix $\xi$ is positive semi-definite by definition. If so, $\mathbf{I}-\gamma \mathbf{R}_{y y}^{-1} \mathbf{R}_{x x}$ must be too.

Thus, using the Eigen-Decomposition of $\mathbf{R}_{y y}^{-1} \mathbf{R}_{x x}$ defined by $\mathbf{U} \Lambda \mathbf{U}^{H}$,

$$
\mathbf{I}-\gamma \mathbf{R}_{y y}^{-1} \mathbf{R}_{x x} \succcurlyeq 0 \Rightarrow \mathbf{I}-\gamma \mathbf{U} \Lambda \mathbf{U}^{H} \succcurlyeq 0 \Rightarrow \mathbf{I}-\gamma \Lambda \succcurlyeq 0 \Rightarrow \Lambda^{-1}-\gamma \mathbf{I} \succcurlyeq 0
$$

where $\Lambda^{-1}$ is a diagonal matrix whose diagonal elements are the corresponding eigenvalues of the matrix $\left(\mathbf{R}_{x x}^{-1} \mathbf{R}_{y y}\right)$. In the worst case we assume that the minimum eigenvalue of $\left(\Lambda^{-1}-\gamma \mathbf{I}\right)$ is equal to zero,

$$
\lambda_{\min }\left(\mathbf{R}_{x x}^{-1} \mathbf{R}_{y y}\right)-\gamma=0
$$

Therefore, another possible estimator of $\gamma$ can be obtained as,

$$
\gamma_{M}=\lambda_{\min }\left(\mathbf{R}_{x x}^{-1} \mathbf{R}_{y y}\right)=\lambda_{\min }\left(\left(\Phi \mathbf{R}_{c m} \Phi^{H}\right)^{-1} \hat{\mathbf{R}}_{y}\right)
$$

The last procedure is denoted as CANDIDATE-M because it looks for the minimum eigenvalue of $\left.\left(\Phi \mathbf{R}_{c m} \Phi^{H}\right)^{-1} \hat{\mathbf{R}}_{y}\right)$.

\section{Numerical results}

This section is divided in two parts. The first part concentrates on the general performance of the candidate spectrum sensing method proposed in the previous section. In the first section, scenarios with high SNR are used for the sake of figure clarity. The second part gives the ROC results for low SNR scenarios.

\subsection{High SNR scenario}

To test the ability of the proposed sparse correlation matching-based spectrum sensing techniques to properly label a desired user, we first consider a scenario with one desired user in the presence of noise. The desired user is assumed to be a binary phase shift keying (BPSK) signal with a rectangular pulse shape, and 4 samples per symbol. The SNR of the desired user is $10 \mathrm{~dB}$ and the normalized carrier frequency is $w_{0}=0.2$. The size of the observation $\mathbf{x}_{m}$ in $L=33$ samples. The sampling rates of $\mathbf{y}_{m}$ and $\mathbf{x}_{m}$ are related through the compression rate $\rho=\frac{p}{L}$. To strictly focus on the performance behavior due to compression and remove the effect of insufficient data records, the size of the compressed observations is forced to be the same for any compression rate. Therefore, we set $M=2 L \epsilon \rho^{-1}$ where $\epsilon$ is a constant (in the following results $\epsilon=10$ ). Thus, for a high compression rate, the estimator takes samples for a larger period of time. The spectral occupancy $\Omega$ for this particular example is 0.25 . The simulation parameters are summarized in Table 1.

Figure 4 shows the performance of the three Candidate methods for different compression rates. The $\gamma_{F}$ estimate, which is shown in Figure 4a, presents lower resolution and higher leakage compared with $\gamma_{M}$ and $d_{\text {geo, } \min }^{-1}$, which are plotted in Figures $4 \mathrm{~b}$ and $4 \mathrm{c} 1$, respectively. From Figure 4 it can be concluded that the best power estimate in terms of resolution is given by $\gamma_{M}$. Moreover, the range of $d_{g e o, m i n}^{-1}$ is smaller than the range of $\gamma_{M}$, which is longer than $15 \mathrm{~dB}$ when there is no compression and decreases when the compression rate increases. This robustness makes us think that CANDIDATE-M may still work in scenarios with low SNR, where CANDIDATE-G probably fails (it is confirmed in the following section). On the other hand, the independence of $\gamma_{G}$ with respect to the carrier frequency may be observed in Figure 4c2.

Figure 5 depicts the same as Figure 4 but with two BPSK desired signals: one located at normalized frequency 0.2 and one located at normalized frequency 0.7 with the same SNR level equal to $10 \mathrm{~dB}$. While all three methods successfully detect the two candidates, $\gamma_{G}$ is not able to provide two power level estimates because of the non-dependency on the frequency of the parameter $\gamma_{G}$.

Figure 6 depicts the same as Figure 4 but with the presence of a narrow band interference (pure tone) with SNR $=10 \mathrm{~dB}$ at normalized frequency 0.7 . While $\gamma_{M}$ remains practically unfazed, CANDIDATE-G's performance has suffered a slight degradation. CANDIDATEF clearly works as a ED. This sensitivity to interference suggests to discard CANDIDATE-F in favor of the two other candidate methods. Although both figures (Figures 4 and 6) make evident the degradation of the correlation-matching based spectrum sensing techniques in terms of detection capability due to the effect of the compression, it is interesting to note that the frequency and power level estimation do not suffer from the compression.

For the evaluation of the frequency and power estimation accuracy, an scenario with one active primary user with binary phase shift keying (BPSK) using a rectangular pulse shape (with 4 samples per symbol) and AWGN is considered. The normalized carrier frequency is again $w_{0}=0.2$ and the size of the observation $\mathbf{x}_{m}$ in $L=33$ samples. Figures 7 and 8 show the normalized root mean squared error (RMSE) of the estimated power level (this is the RMSE divided by the SNR) and the normalized RMSE of the estimated frequency location

Table 1 Simulation parameters

\begin{tabular}{ccccc}
\hline$\rho=\frac{p}{L}$ & $\mathbf{1}$ & $\mathbf{0 . 7 6}$ & $\mathbf{0 . 5 2}$ & $\mathbf{0 . 2 4}$ \\
\hline$Q$ & 33 & 25 & 17 & 8 \\
$M$ & 660 & 871 & 1281 & 2723 \\
Acquisition time (ms) & 2.2 & 2.9 & 4.2 & 9.0 \\
\hline
\end{tabular}




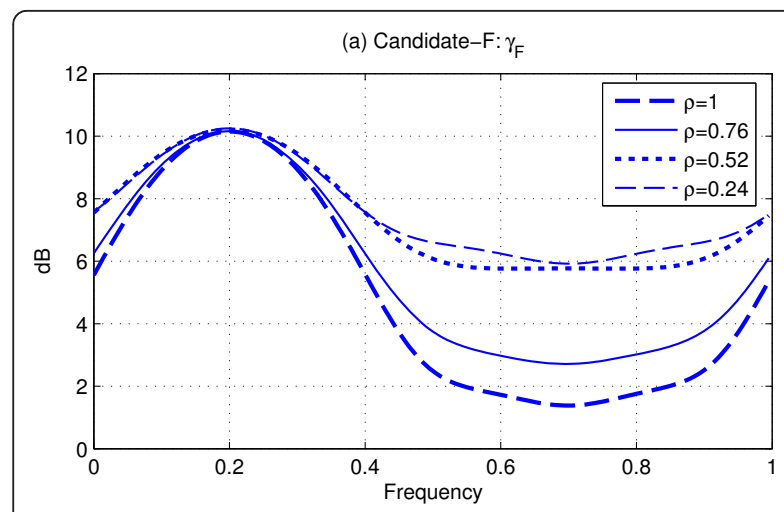

(c1) Canidate-G: $\mathrm{d}_{\text {geo,min }}^{-1}$

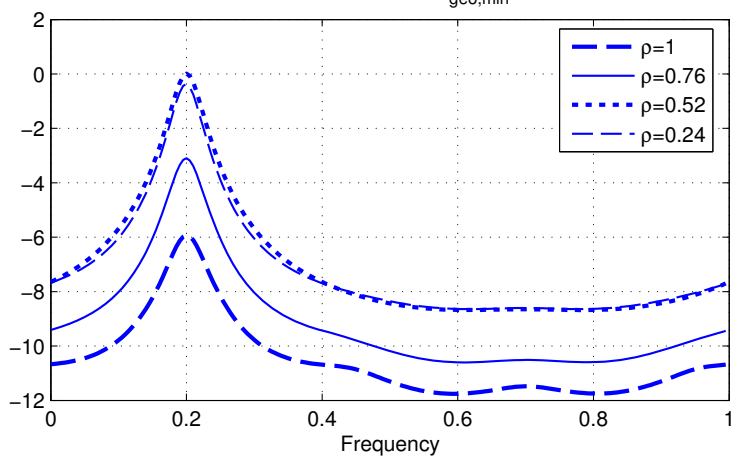

(b) Canidate-M: $\gamma_{M}$

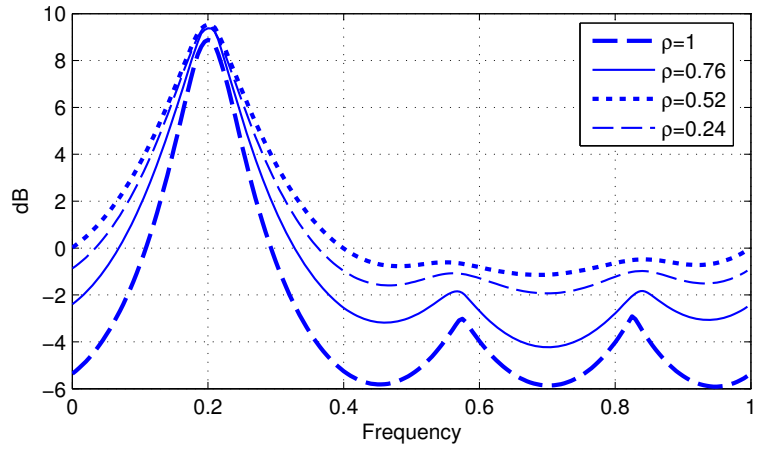

(c2) Canidate-G: $\gamma_{G}$

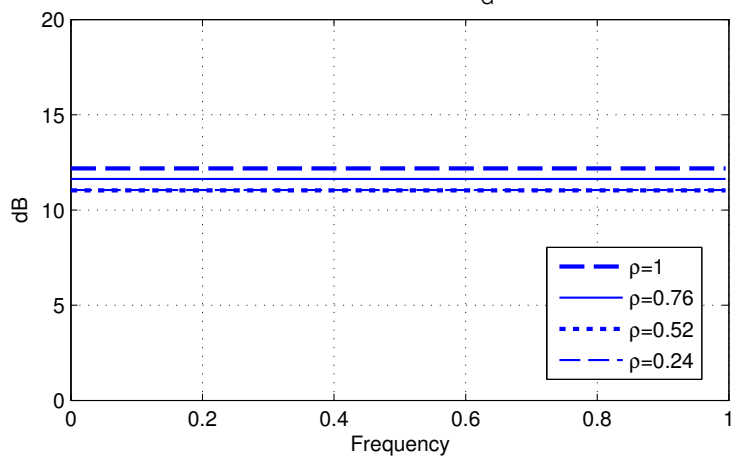

Figure 4 Performance of the sparse correlation-matching based spectrum sensing. The candidate has the following parameters: BPSK signal with 4 samples per symbol, and SNR $=10 \mathrm{~dB}$ at frequency 0.2 .

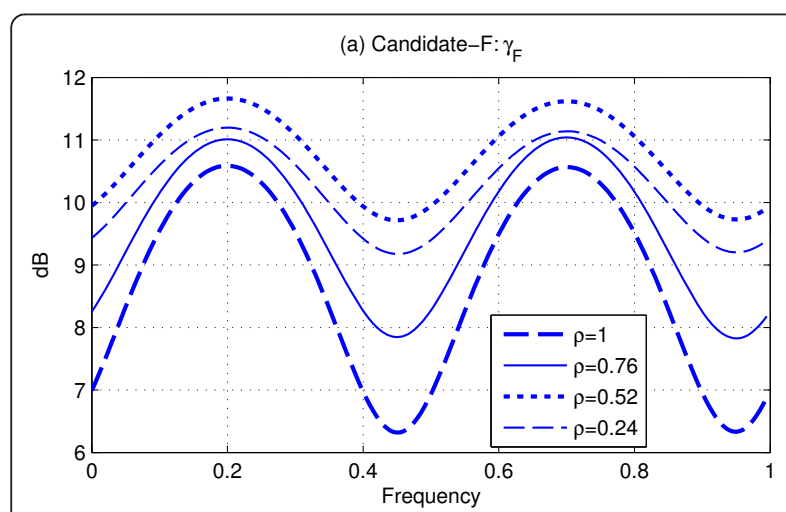

(c1) Canidate-G: $\mathrm{d}_{\mathrm{geo}, \mathrm{min}}^{-1}$

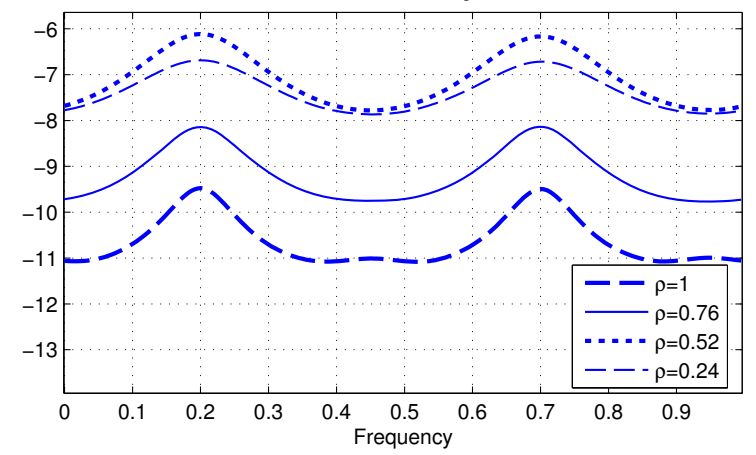

(b) Canidate-M: $\gamma_{M}$

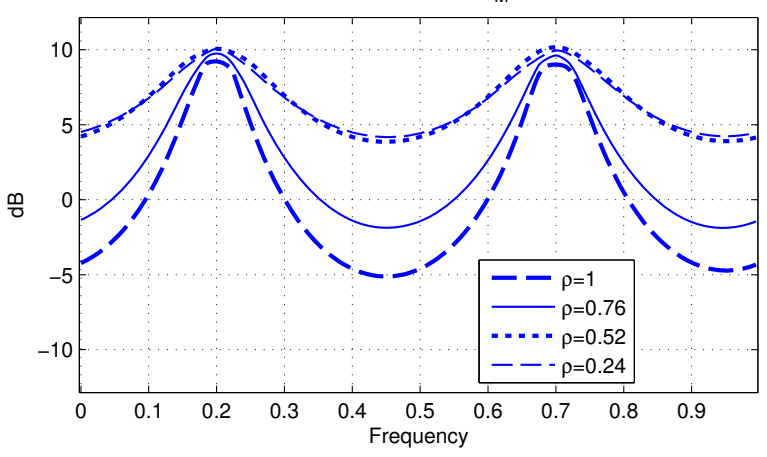

(c2) Canidate-G: $\gamma_{G}$

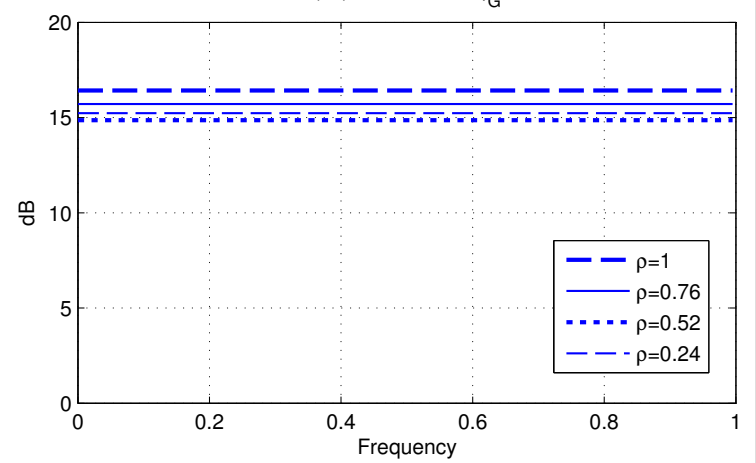

Figure 5 Performance of the sparse correlation-matching based spectrum sensing. Two desired users: both BPSK signals with 4 samples per symbol, and SNR $=10 \mathrm{~dB}$ at frequency 0.2 and 0.7 . 


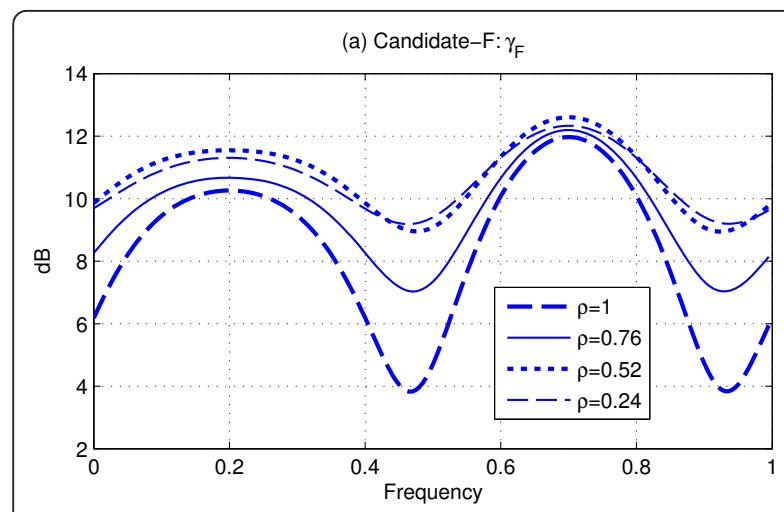

(c1) Canidate-G: $d_{\text {geo,min }}^{-1}$

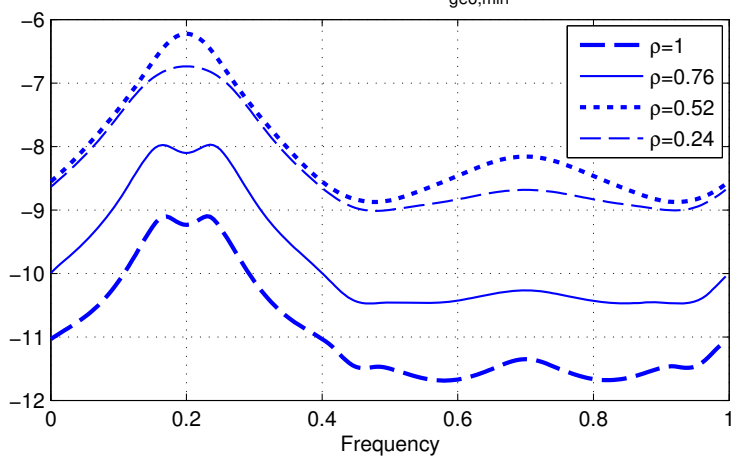

(b) Canidate-M: $\gamma_{\mathrm{M}}$

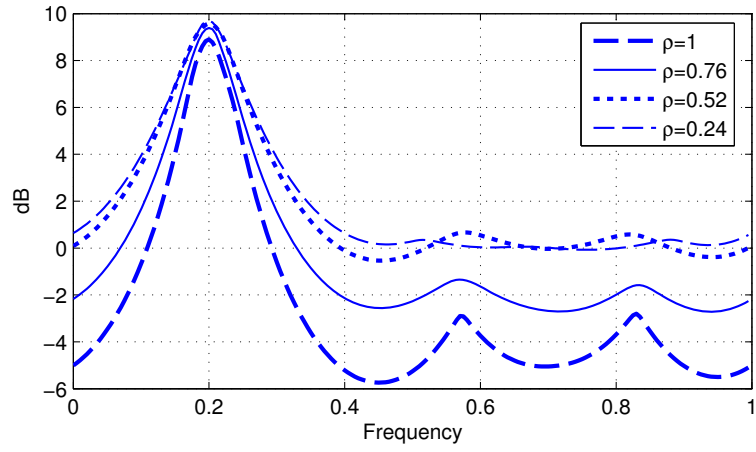

(c2) Canidate-G: $\gamma_{G}$

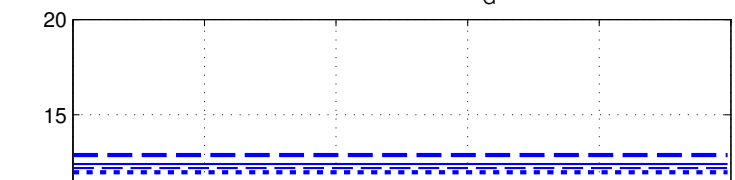

$\cong 10$

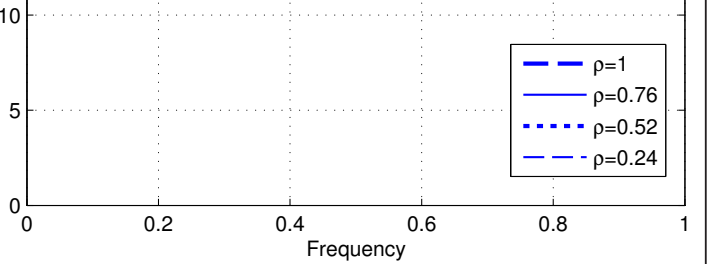

Figure 6 Performance of the sparse correlation-matching based spectrum sensing with interference. The candidate has the following parameters: BPSK signal with 4 samples per symbol, and SNR $=10 \mathrm{~dB}$ at frequency 0.2. The interference is a pure sinusoid located at frequency 0.7 and with $\mathrm{SNR}=10 \mathrm{~dB}$.

(this is the RMSE divided by $w_{0}$ ) of the desired user, respectively, for different compression rates. From Figures 7 and 8 it can be conclude that both the power level estimation accuracy and the frequency estimation accuracy remain almost constant whatever the compression rate we consider.

On the other hand, Figure 9 shows the comparison between CANDIDATE-M and CANDIDATE-G for a
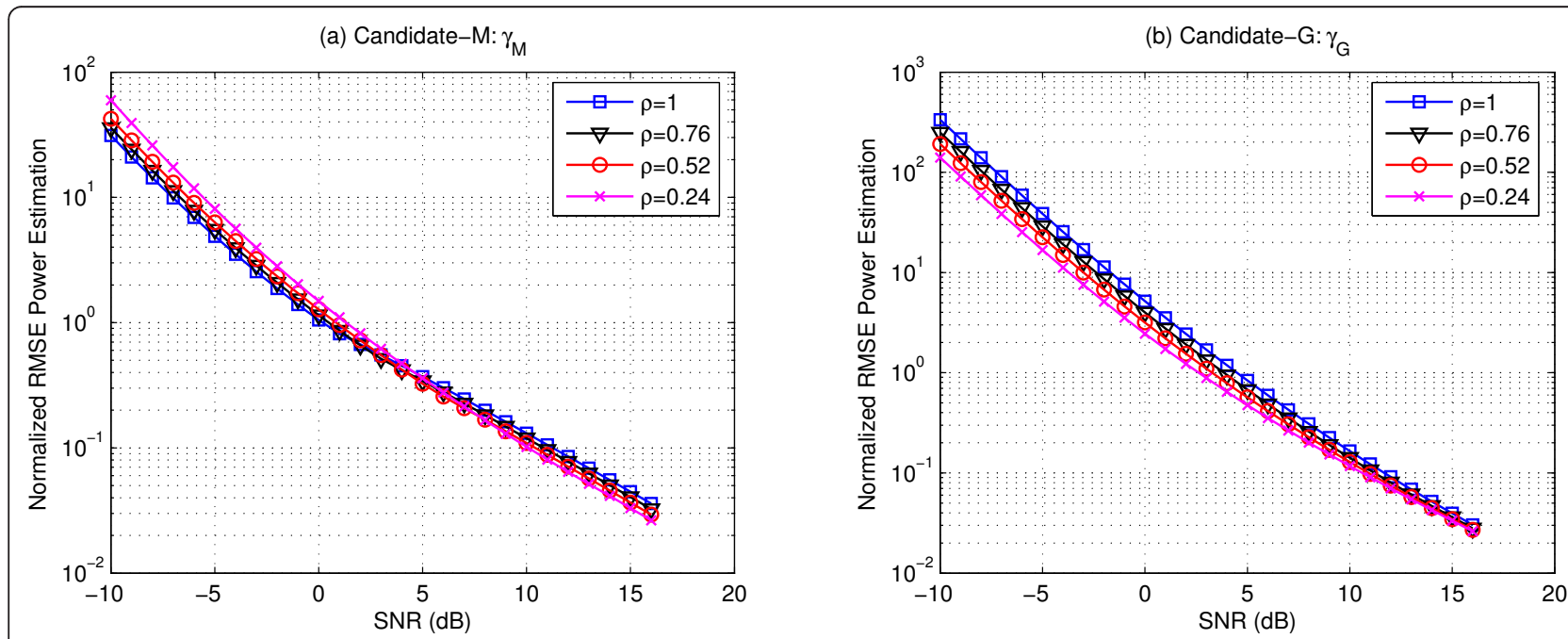

Figure 7 RMSE of the estimated power level in a scenario with one desired user (BPSK) in the presence of noise. (a) CANDIDATE-M, (b) CANDIDATE-G 

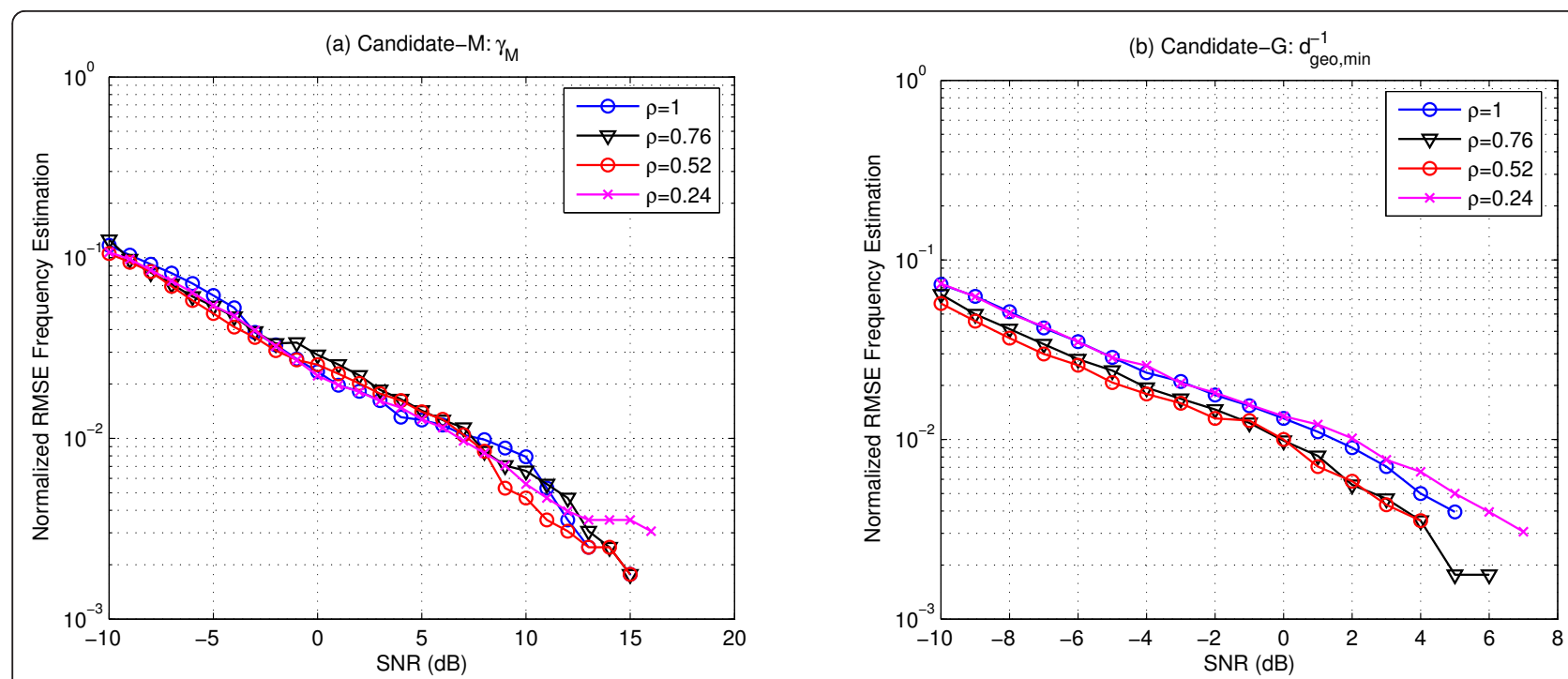

Figure 8 RMSE of the estimated frequency location in a scenario with one desired user (BPSK) in the presence of noise. (a) CANDIDATE-M, (b) CANDIDATE-G

particular compression rate in terms of RMSE. Figure 9a reflects that CANDIDATE-M presents higher resolution in the power estimation than CANDIDATE-G. However, $\gamma_{G}$ does not depend on the frequency and therefore it only works properly when only one desired user is present. Moreover, Figure 9b makes evident that CANDIDATE-G provides better results for the frequency location estimation. In conclusion, CANDIDATE-M seems to be the most complete technique of the three proposed methods because it provides good frequency and power estimations and it works in scenarios where more than one desired user is present.
The results obtained in Figure 6 can be compared with the performance of the current most prominent spectral estimation procedures applied in the scenario under consideration. To this end, Figure 10 shows two major filter-bank spectral estimates (periodogram and normalized capon) and Thompson's mul-titaper method (MTM) [26] for the scenario with interference in 0.7. Besides presenting low resolution, note also that they are not robust to the strong interference. In contrast, the candidate methods provide a clear frequency and power estimation and make the interference disappear because of their feature-based nature.
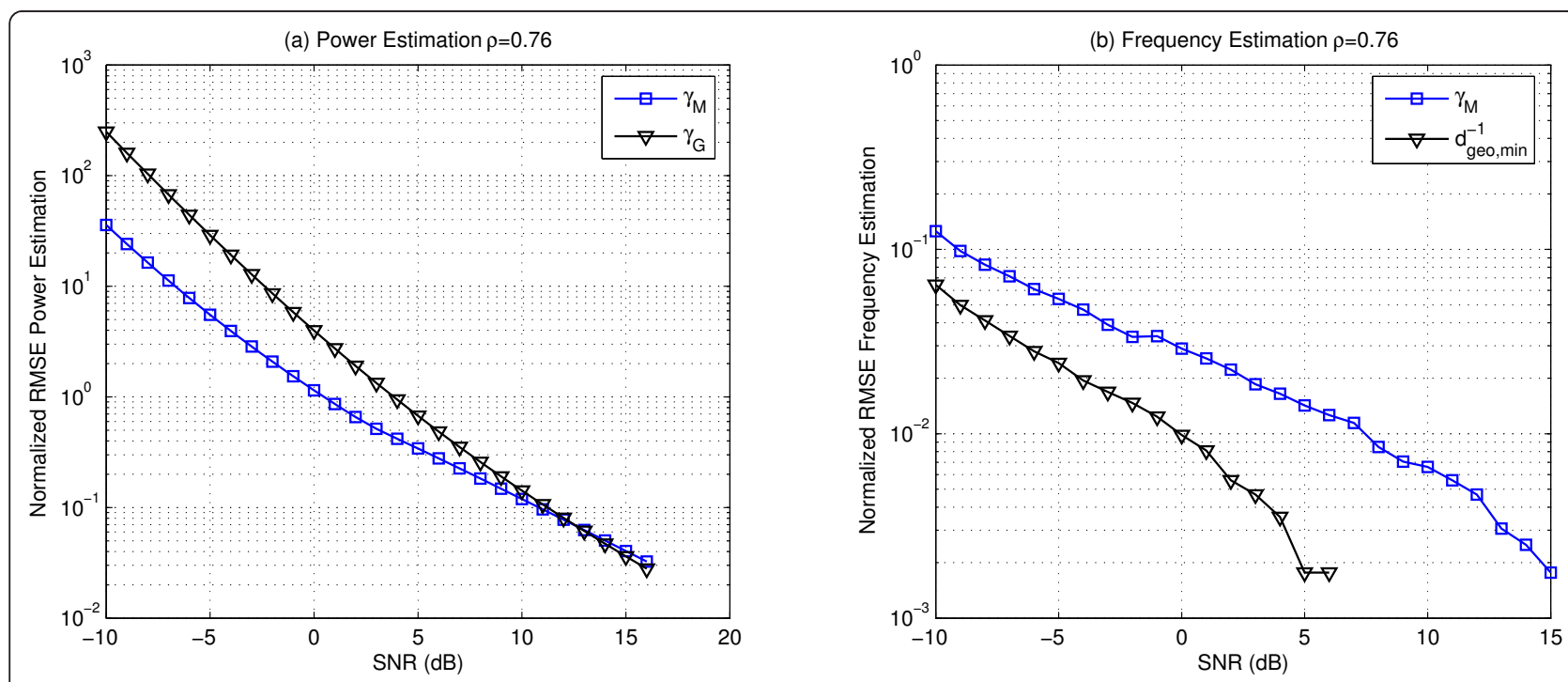

Figure 9 RMSE of the estimated parameters $(\rho=0.76)$. Performance comparison of CANDIDATE-M and CANDIDATE-G. (a) RMSE of the power level estimation. (b) RMSE of the frequency location estimation. 


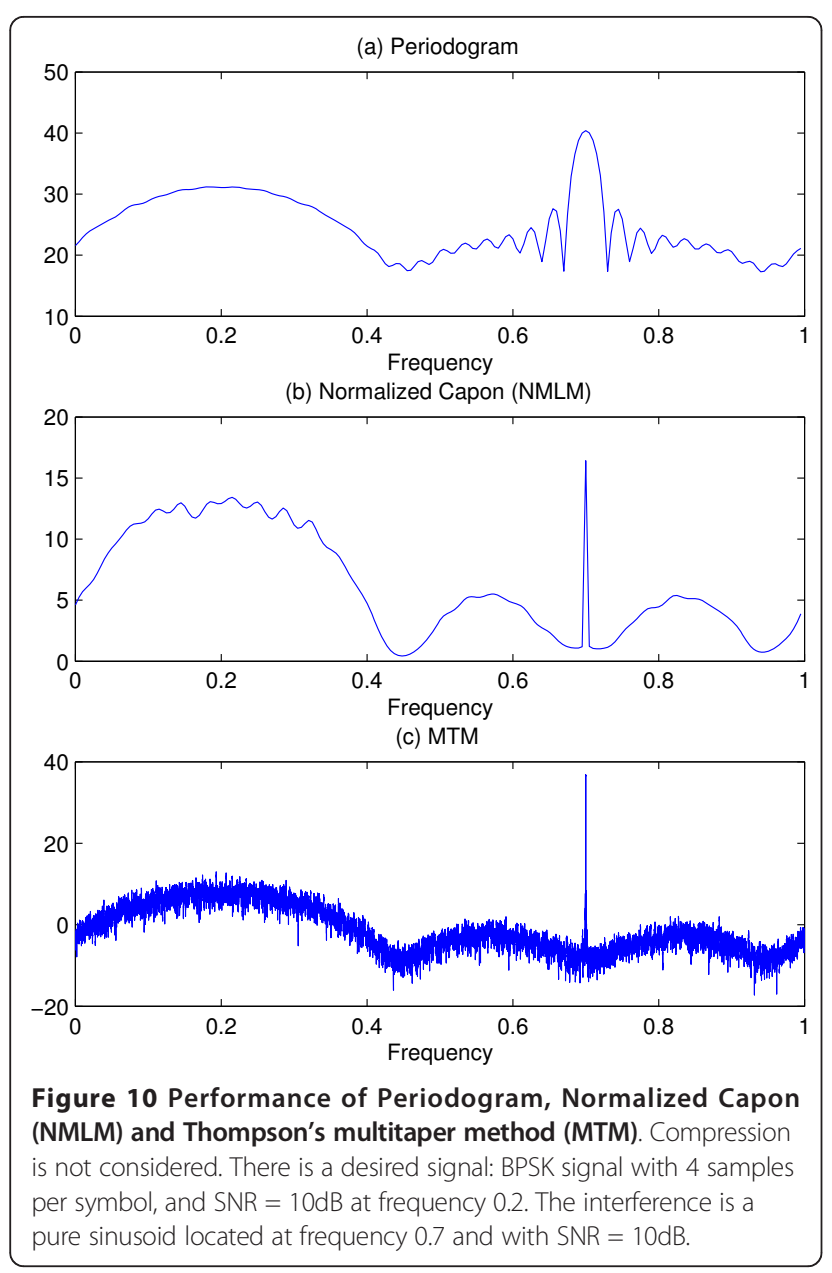

Because the method proposed here is a correlation matching-based method, a question regarding its robustness in front of a frequency selective channel, instead of flat fading, arises. We consider here the same scenario as in Figure 6 but with a 13-tap Rice channel (a channel with a LOS and some random late arrivals). The LOS presents a gain of one, and the late random arrivals altogether also have energy equal to one. A particular performance is shown in Figure 11 for $\rho=0.76$ and in Figure 12 for $\rho=0.52$. It can be observed that the multipath causes significant losses regarding detection capabilities and also a deterioration in the frequency estimation due to the appearance of a bias into the frequency location. In any case, if these losses imply serious problem, it is reasonable to assume channel equalization at the sensing station.

\subsection{Low SNR scenario-ROC curves}

This section evaluates the performance in low SNR scenarios by means of the ROC curves (receiver operating characteristic) in order to illustrate the proposed candidate spectrum sensing method robustness against noise.

To evaluate the probability of false alarm versus the probability of detection we have run 200 simulations, each in the presence of the primary user (Hypothesis $\mathrm{H} 1$ ), and 200 records of the same length without the primary user (Hypothesis H0).

$$
\hat{\mathbf{R}}_{y}=\left\{\begin{array}{lr}
\mathbf{R}_{n} & H_{0} \\
\gamma\left(w_{s}\right) \Phi \mathbf{R}_{c m} \Phi^{H}+\mathbf{R}_{w} H_{1}
\end{array}\right.
$$
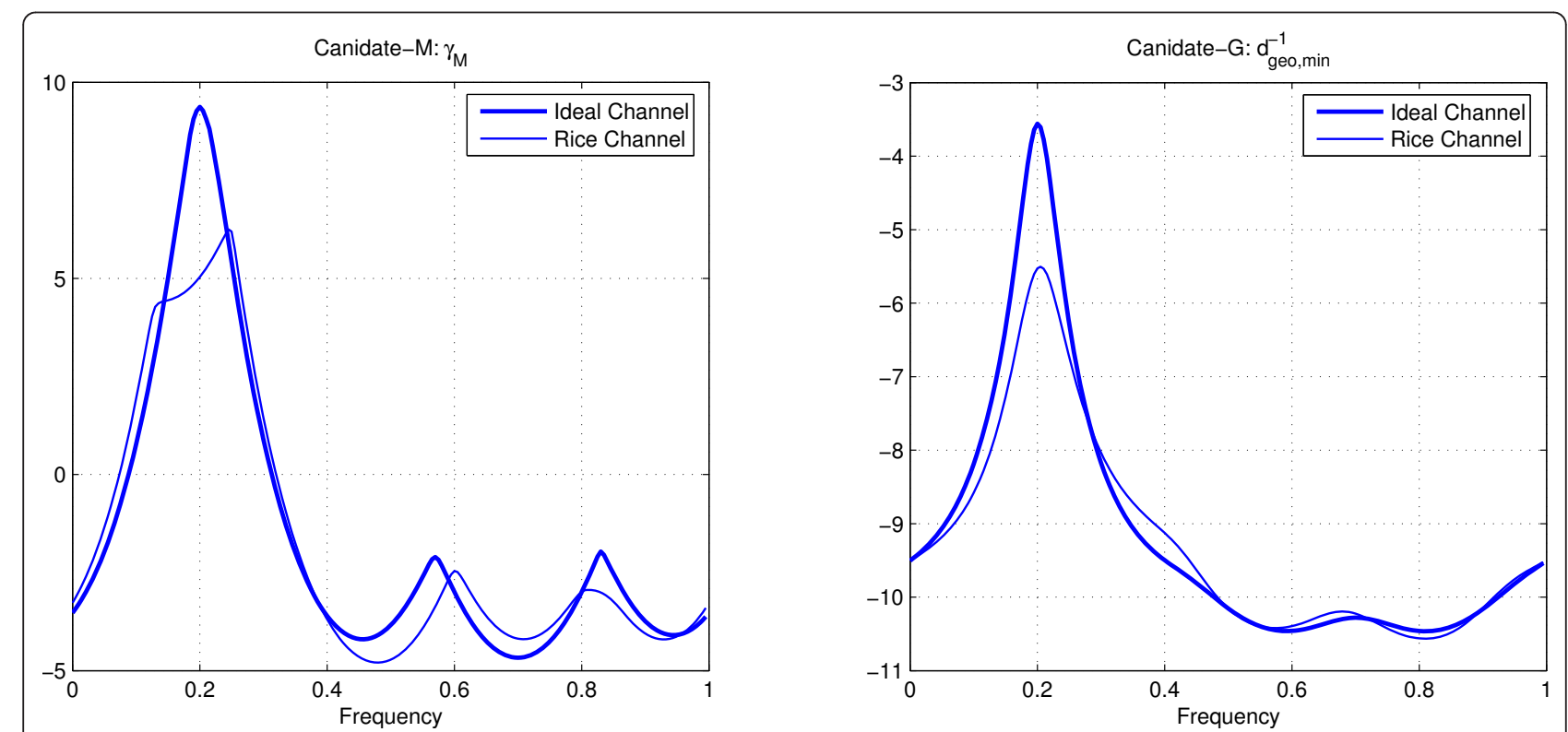

Figure 11 Performance of CANDIDATE-M and CANDIDATE-G in a rice channel $(\boldsymbol{\rho}=\mathbf{0 . 7 6})$. The candidate is a BPSK, with 4 samples per symbol, and $S N R=10 \mathrm{~dB}$ at frequency 0.2. The interference is a pure tone located at 0.3 with $S N R=10 \mathrm{~dB}$. A 13-length rice channel is considered. 


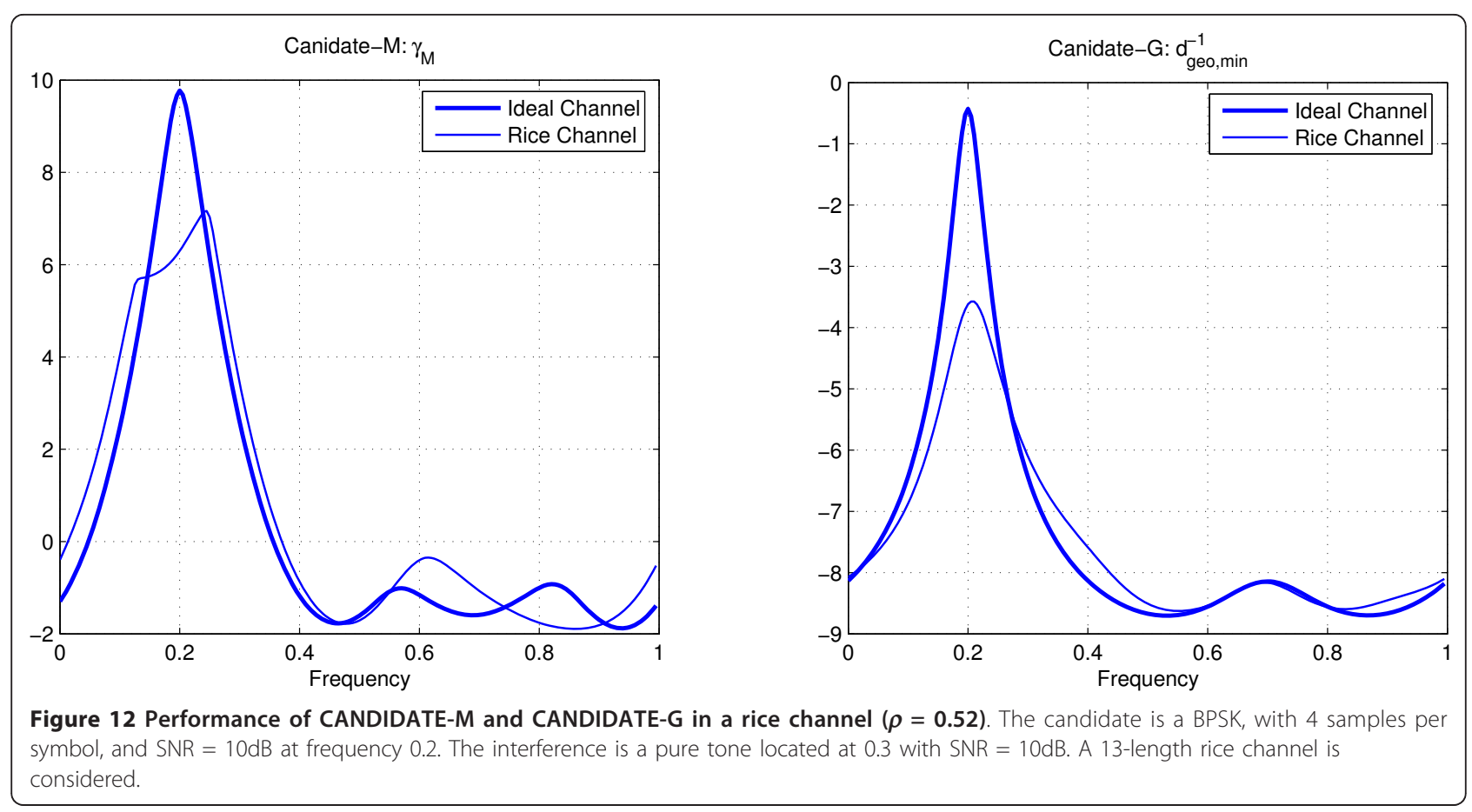

The primary user is located at normalized frequency 0.2. The primary user is a BPSK and its SNR is indicated in each plot. The detection methods under study will be CANDIDATE-M and CANDIDATE-G.

Figure 13a shows the ROC of CANDIDATE-M and Figure 13b the ROC of CANDIDATE-G for SNR $=-10$ $\mathrm{dB}$ obtained with Monte Carlo runs and for different compression rates. Figure 14 shows the same but with $\mathrm{SNR}=-14 \mathrm{~dB}$. As it was expected, the general performance of both detectors is deteriorated as the compression rate decreases. One possible explanation of why we are losing detection capabilities when the compression rate decreases can be found in [27], where the noise folding phenomenon is described. Basically, [27] stated that for the acquisition of a noisy signal of fixed sparsity, the SNR of the CS measurements decreases by $3 \mathrm{~dB}$ for every octave increase in the subsampling factor.

Both Figures 13 and 14 make evident that the CANDIDATE-M performance is much better than that of CANDIDATE-G.

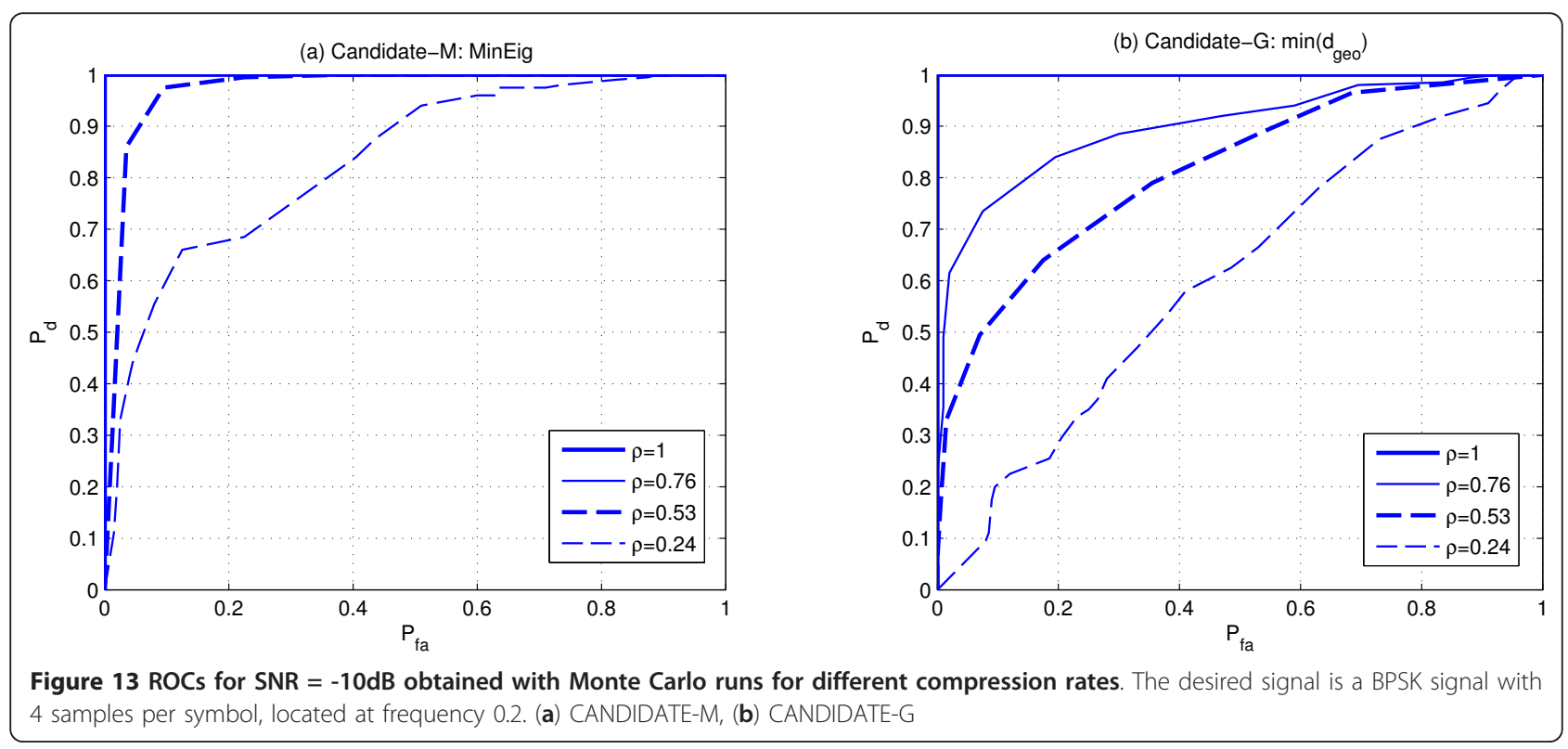



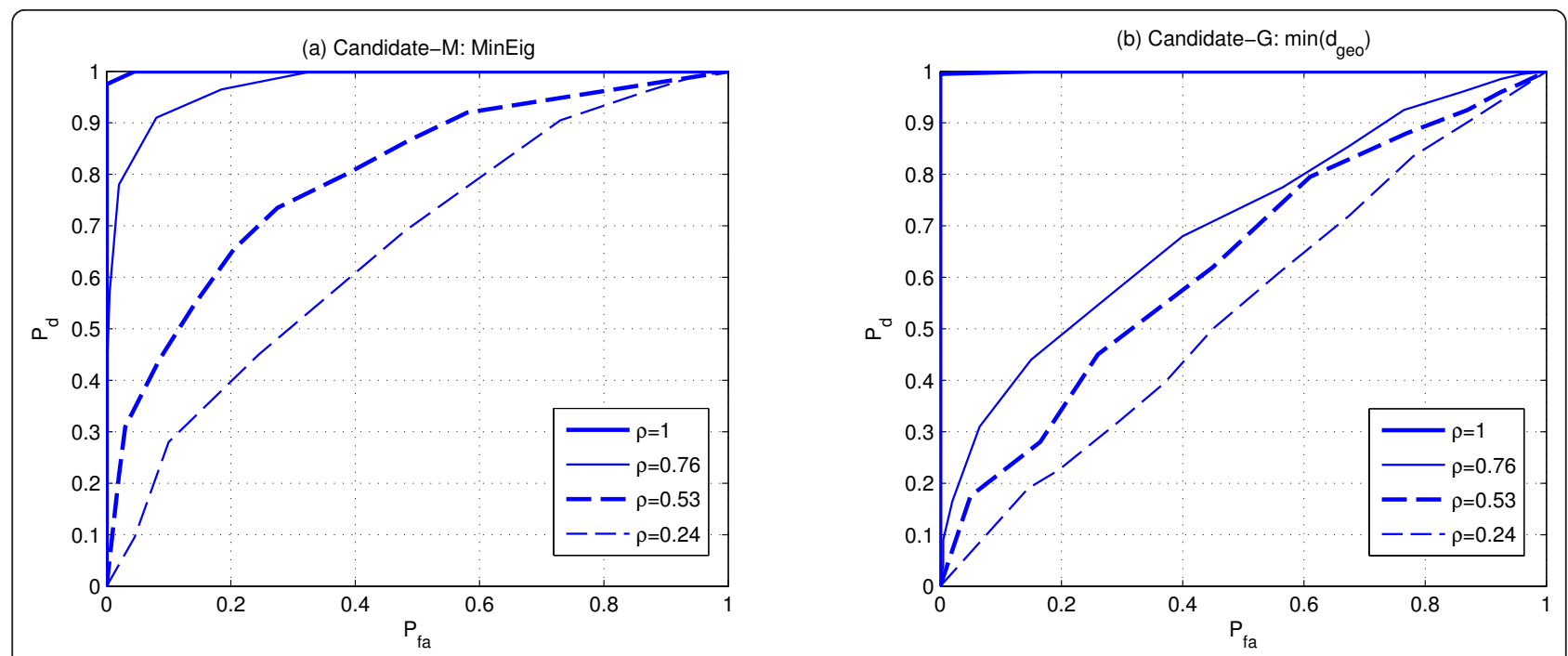

Figure 14 ROCs for SNR $=-14 \mathrm{~dB}$ obtained with Monte Carlo runs for different compression rates. The desired signal is a BPSK signal with 4 samples per symbol, located at frequency 0.2. (a) CANDIDATE-M, (b) CANDIDATE-G

\section{Summary and conclusions}

A feature-based approach for spectrum sensing based on periodic non-uniform sampling is addressed. In particular, the compressed-sampling version of detecting predetermined spectral shapes in sparse wideband regimes is faced by means of a correlation-matching procedure. The main contribution of the new subNyquist sampling approach is that it allows to alleviate the amount of data needed in the spectrum sensing process. Once the sampling bottleneck is solved, the data autocorrelation matrix is obtained from subNyquist samples. Following the correlation matching concept, the method is able to provide an estimate of the frequency location and a power level estimation of the desired user. Three different methods are proposed: The first one, which is based on the Euclidean distance, is discarded because of its low rejection to interference. The second one, which is based on the geodesic distance, works well in terms of interference rejection but the power level estimate that this method provides does not depend on the frequency parameter and therefore, it is not indicated when detecting more than one desired user. The third method, which is based on the positive semidefinite difference between matrices, is the one which works the better, both in terms of accuracy of the estimated parameters and in terms of robustness against noise. As it was expected, simulation results have shown that the compression affects the detection capabilities of all the correlation-matching methods. However, we have also shown that the accuracy of the frequency estimation and the accuracy of the power level estimation is not affected by the undersampling technique.

\section{Acknowledgements}

This study was partially supported by the Catalan Government under grant 2009 SGR 891, by the Spanish Government under project TEC2008-06327-C03 (MULTI-ADAPTIVE), by the European Cooperation in Science and Technology under project COST Action IC0902. Eva Lagunas is supported by the Catalan Government under grant FI-DGR 2011.

\section{Endnotes}

ahttp://www.fcc.gov/.

bhttp://www.etsi.org/.

\section{Author details}

'Department of Signal Theory and Communications, Technical University of Catalonia (UPC), c/Jordi Girona 1-3, 08034, Barcelona, Spain ${ }^{2}$ Centre

Tecnologic de Telecomunicacions de Catalunya (CTTC), Av. Canal Olímpic SN, 08860, Castelldefels, Spain

\section{Competing interests}

The authors declare that they have no competing interests.

Received: 15 September 2011 Accepted: 15 February 2012 Published: 15 February 2012

\section{References}

1. Federal Communications Commission, Tech. Report TR 02-155 (November 2002)

2. J Mitola III, GQ Maguire Jr, Cognitive radio: making software radios more personal. IEEE Personal Commun. 6(4), 13-18 (1999). doi:10.1109/98.788210

3. D Cabric, SM Mishra, RW Brodersen, Implementation issues in spectrum sensing for cognitive radios. Asilomar Conf Signals Syst Comput. 1, 772-776 (2004)

4. JA Tropp, JN Laska, MF Duarte, JK Romberg, RG Baraniuk, Beyond Nyquist: efficient sampling of sparse bandlimited signals. IEEE Trans Inf Theory 56(1), 520-544 (2010)

5. CE Shannon, Communication in the presence of noise. Proc Inst Radio Eng. 37(1), 10-21 (1949) 
6. H Nyquist, Certain topics in telegraph transmission theory. Trans AIEE. 47, 617-644 (1928)

7. HS Shapiro, RA Silverman, Alias-free Sampling of random noise. J Soc Indust Appl Math. 8, 225-248 (1960). doi:10.1137/0108013

8. FJ Beutler, Alias-free randomly timed sampling of Stochastic processes. IEEE Trans Inf Theory 16, 147-152 (1970). doi:10.1109/IIT.1970.1054435

9. E Masry, Alias-free sampling: an alternative conceptualization and its applications. IEEE Trans Inf Theory. 24(3), 317-324 (1978). doi:10.1109/ TIT.1978.1055889

10. I Bilinskis, AK Mikelsons, Randomized Signal Processing, (Prentice Hall, London, 1992)

11. I Bilinskis, Digital Alias-free Signal Processing, (Wiley, Chichester, UK, 2007)

12. P Feng, Y Bresler, Spectrum-blind minimum-rate sampling and reconstruction of multiband signals, Inter Conf Acoustics Speech Signal Process, 3 Atlanta, GA, 1688-1691 (1996)

13. DL Donoho, Compressed sensing. IEEE Trans Inf Theory 52(4), 1289-1306 (2006)

14. EJ Candes, MB Wakin, An introduction to compressed sampling. IEEE Signal Process Mag. 25(2), 21-30 (2008)

15. M Mishali, Y Eldar, O Dounaevsky, E Shoshan, Xampling: analog to digital at sub-Nyquist rates. IET J Circ Dev Syst. 5(1), 8-20 (2011). doi:10.1049/ietcds. 2010.0147

16. Z Tian, GB Giannakis, Compressed sensing for wideband cognitive radios, International Conference on Acoustics, Speech and Signal Processing (ICASSP), 4 Las Vegas, NV, 1357-1360 (2008)

17. H Urkowitz, Energy detection of unknown deterministic signals. IEEE Proc. 55, 523-531 (1967)

18. W Gardner, Signal interception: a unifying theoretical framework for feature detection. IEEE Trans Commun. 36, 897-906 (1988). doi:10.1109/26.3769

19. T Yucek, H Arslan, A survey of spectrum sensing algorithms for cognitive radio applications. IEEE Commun Surv Tutorials. 11(1), 116-130 (2009)

20. YL Polo, Y Wang, A Pandharipande, G Leus, Compressive wide-band spectrum sensing, in International Conference on Acoustics, Speech and Signal Processing (ICASSP), Taipei, 2337-2340 (2009)

21. Y Wang, Z Tian, C Feng, A two-step compressed spectrum sensing scheme for wideband cognitive radios, in IEEE Global Telecommunications Conference (GLOBECOM), Miami, FL, 1-5 (2010)

22. M Rashidi, K Haghighi, A Owrang, M Viberg, A wideband spectrum sensing method for cognitive radio using sub-Nyquist sampling, in IEEE Signal Processing Society 14th DSP Workshop, 6th SPE Workshop, Arizona, USA 30-35 (2010)

23. MA Lexa, ME Davies, JS Thompson, J Nikolic, Compressive power spectral density estimation, in International Conference on Acoustics, Speech and Signal Processing (ICASSP), Prague, 3884-3887 (2011)

24. Al Pérez-Neira, MA Lagunas, MA Rojas, P Stoica, Correlation matching approach for spectrum sensing in open spectrum communications. IEEE Trans Signal Process. 57(12), 4823-4836 (2009)

25. P Feng, Universal minimum-rate sampling and spectrum-blind reconstruction for multiband signals, PhD, (University of Illinois at UrbanaChampaign, USA, 1997)

26. PD Welch, The use of FFT for the estimation of power spectra: A method based on time averaging over short modified periodograms. IEEE Trans Audio Elec-troacustic. AU-15(2), 70-73 (1967)

27. J Treichler, M Davenport, R Baraniuk, Application of compressive sensing to the design of wideband signal acquisition receivers, in Proc 6th US and Australia Joint Workshop on Defense Applications of Signal Processing (DASP), (Hawaii, 2009)

doi:10.1186/1687-6180-2012-31

Cite this article as: Lagunas and Nájar: Sparse correlation matchingbased spectrum sensing for open spectrum communications. EURASIP Journal on Advances in Signal Processing 2012 2012:31.

\section{Submit your manuscript to a SpringerOpen ${ }^{\circ}$ journal and benefit from:}

- Convenient online submission

- Rigorous peer review

- Immediate publication on acceptance

- Open access: articles freely available online

- High visibility within the field

- Retaining the copyright to your article

Submit your next manuscript at $\gg$ springeropen.com 